\title{
Composite resonances and their impact on the electroweak chiral Lagrangian
}

\author{
Juan José Sanz-Cillero* ${ }^{*}$ \\ Departamento de Física Teórica and Instituto de Física Teórica, IFT-UAM/CSIC, \\ Universidad Autónoma de Madrid, Cantoblanco, 28049 Madrid, Spain \\ E-mail: juanj.sanz@uam.es
}

In this talk we study the low-energy effective couplings generated by strongly-coupled electroweak models that contain heavy composite resonances. Invariance under $S U(2)_{L} \times S U(2)_{R}$ is a key ingredient in the construction of the resonance action. For simplicity, in these proceedings we focus our attention on the impact of a heavy colourless vector $V$, which transforms as a triplet under the custodial group. More precisely, we study the couplings that are relevant for the vector form-factors of the $L+R$ current into two electroweak Goldstones and into two Standard Model fermions, which contribute to the oblique parameters $S$ and $T$ and the anomalous $Z \rightarrow f \bar{f}$ couplings, respectively. Our predictions are compatible with bounds from direct and indirect searches for $M_{V} \gtrsim 1.5 \mathrm{TeV}$. Finally, although we consider an antisymmetric tensor formalism to describe the vector resonance, we derive the equivalent action in the Proca four-vector representation and show that the predictions for low-energy couplings and form-factors are identical, as expected.

The European Physical Society Conference on High Energy Physics

22-29 July 2015

Vienna, Austria

\footnotetext{
* Speaker.

$\dagger$ I would like to thank the organizers for the nice scientific and social environment during the Conference. Lots of thanks also to A. Pich, I. Rosell and J. Santos for their help in the preparation of these proceedings. This work has been supported by ERDF funds from the European Commission [FPA2013-44773-P, SEV-2012-0249, CSD2007-00042].
} 


\section{Impact of heavy resonances on the low-energy electroweak effective theory}

So far the Large Hadron Collider (LHC) has not found any trace of beyond the Standard Model (BSM) states with masses below $1 \mathrm{TeV}$. Likewise, no significant deviation has been observed in the low-energy interactions between Standard Model (SM) particles. Effective field theories are then the natural approach. In this talk $[1,2]$ we discuss the possibility of strongly-coupled BSM scenarios with the approximate custodial symmetry invariance of the SM, exact in the SM scalar sector. We develop an invariant Lagrangian under $\mathscr{G}=S U(2)_{L} \times S U(2)_{R}$, which spontaneously breaks down to the custodial subgroup $\mathscr{H}=S U(2)_{L+R}$ and generates the electroweak (EW) wouldbe Goldstone bosons $\varphi^{a}$, described a unitary $2 \times 2$ matrix $U(\varphi)$. In these (non-linear) EW chiral Lagrangian with a light Higgs (ECLh), the low-energy amplitude $\mathscr{M}$ has an expansion in powers of infrared scales $p$ (external momenta and SM masses) of the form (e.g., for $2 \rightarrow 2$ processes) [2, $3,4,5,6]$,

$$
\mathscr{M} \sim \underbrace{\frac{p^{2}}{v^{2}}}_{\text {LO (tree) }}+(\underbrace{a_{k}^{r}}_{\text {NLO (tree) }}-\underbrace{\frac{\Gamma_{k}}{16 \pi^{2}} \ln \frac{p}{\mu}+\ldots}_{\mathrm{NLO}(1-\text { loop })}) \frac{p^{4}}{v^{4}}+\mathscr{O}\left(p^{6}\right) .
$$

The EW effective theory (EWET) Lagrangian operators can be sorted out based on their chiral dimension:

$$
\mathscr{L}_{\mathrm{EWET}}=\mathscr{L}_{2}+\mathscr{L}_{4}+\ldots
$$

where the operators in $\mathscr{L}_{\hat{d}}$ are of $\mathscr{O}\left(p^{\hat{d}}\right)[2,3,4,5]$. Covariant derivatives and masses are $\mathscr{O}(p)$ [7] and each fermion field scales like $\mathscr{O}\left(p^{1 / 2}\right)$ in naive dimensional analysis (NDA) $[2,4,5,8]$. The $\mathscr{G}_{-}$ invariant operators in $\mathscr{L}_{\text {EWET }}$ are built with the Goldstone tensors $U(\varphi)$, functions $\mathscr{F}_{k}$ of the Higgs singlet $h$, its derivatives $\partial_{\mu_{1}} \ldots \partial_{\mu_{n}} h$, the gauge fields and the SM fermions $\psi[8,9,10,11,12,13]$. From the chiral counting point of view $\mathscr{L}^{\mathrm{SM}}$ would be $\mathscr{O}\left(p^{2}\right)$ but its underlying renormalizable structure makes all $\Gamma_{k}=0$ and ensures the absences of higher-dimension divergences [6,14]. The most important contributions to a given process are given by the operators of lowest chiral dimension. The leading order (LO) contribution is $\mathscr{O}\left(p^{2}\right)$ and is given by tree-level diagrams with only $\mathscr{L}_{2}$ vertices. Likewise, the one-loop contribution with only $\mathscr{L}_{2}$ vertices is $\mathscr{O}\left(p^{4}\right)$; it is suppressed in (1.1) with respect to the LO by a factor $p^{2} / \Lambda_{\mathrm{NL}}^{2}$, with $\Lambda_{\mathrm{NL}}^{2} \sim 16 \pi^{2} v^{2} \Gamma_{k}^{-1} \approx 3 \mathrm{TeV}$ (with $\left.v=\left(\sqrt{2} G_{F}\right)^{-1 / 2} \approx 246 \mathrm{GeV}\right)$. This suppression factor is related to the non-linearity of the ECLh and $\Lambda_{\mathrm{NL}} \rightarrow \infty$ when the Higgs can be embedded in a complex doublet $\Phi$ [6]. ${ }^{1}$

In these proceedings $[1,2]$ we focus our attention on the tree-level next-to-leading order (NLO) contributions. They are $\mathscr{O}\left(p^{4}\right)$ and are provided by tree-level diagrams with one $\mathscr{L}_{4}$ vertex with low-energy coupling $a_{k}$ (LEC) and an arbitrary number of $\mathscr{L}_{2}$ vertices. They get contributions from tree-level heavy resonance exchanges. At low energies, these $\mathscr{O}\left(p^{4}\right)$ terms in (1.1) are typically suppressed with respect to the LO amplitude, $\mathscr{O}\left(p^{2}\right)$, by a factor $a_{k} p^{2} / v^{2} \sim p^{2} / M_{R}^{2}[1,2,15,16]$.

\footnotetext{
${ }^{1}$ Ref. [14] provides a geometrical interpretation in terms of the curvature of metric of the internal weak space of the Higgs. In the flat-space limit one has $\Lambda_{\mathrm{NL}} \rightarrow \infty$. Linear-Higgs scenarios with a complex Higgs doublet $\Phi$ correspond to this case. True "non-linear models" are defined by a non-zero curvature, not by their (non-linear) representation.
} 
At high energies, one must include both the light dof (SM particles) and the possible composite resonances as active degrees of freedom (dof) in the Lagrangian [1, 2, 17]:

$$
\mathscr{L}=\mathscr{L}_{\text {non-res }}+\mathscr{L}_{R},
$$

where $\mathscr{L}_{\text {non-res }}$ contains only SM fields and $\mathscr{L}_{R}$ is the part of the Lagrangian that also contains resonances [1]. The part of the interaction Lagrangian $\mathscr{L}_{R}$ relevant for our analysis of the $\mathscr{L}_{4}$ LECs is given by the terms linear in the resonance fields, $\Delta \mathscr{L}_{R}=R \mathbb{O}_{p^{2}}[\chi, \psi][1,2,15,16,17]$, with $\chi(\psi)$ referring to the light bosonic (fermionic) fields. The tensor $\mathbb{O}_{p^{2}}[\chi, \psi]$ that couples the heavy resonance $R$ to the light dof is going to provide the first correction to the low-energy ECLh by means of diagrams where one has a heavy resonance propagator $\sim 1 / M_{R}^{2}$ exchanged between two vertices with $\mathbb{O}_{p^{2}}[\chi, \psi]$. This gives an EWET operator of $\mathscr{O}\left(p^{4}\right)$. At low energies, resonance operators with tensors $\mathbb{O}[\chi, \psi]$ of a higher order in $p$ or containing two or more $R$ fields contribute only to $\mathscr{L}_{\hat{d}}$ with $\hat{d}>4$.

The tree-level contribution to $\mathscr{L}_{\mathrm{EWET}}[\chi, \psi]$ is given by the underlying high-energy action $S[\chi, \psi, R]$ with the resonance fields $R$ evaluated at the classical solution $R_{\mathrm{cl}}(\chi, \psi)$ of their equations of motion (EoM). Solving the resonance EoM and expanding their solutions in powers of momenta for $p \ll M_{R}$, one can write the heavy fields as local operators of the EWET dof [15]. This prediction for the contribution to the low-energy ECLh can be complemented through the consideration of ultraviolet-completion hypotheses (sum-rules [18, 19], unitarity [16], asymptotic form-factor counting rules [20]...). This imposes constraints on the resonance couplings that then turn into predictions for the low-energy theory.

\section{Phenomenological example: vector form-factors}

Let us illustrate this with a basic example. We consider a colourless triplet vector resonance $V$ in a composite theory with the same symmetries of the scalar sector of the SM -invariance under parity and charge conjugation-, with its high energy interaction provided by the Lagrangian [1, 2],

$$
\Delta \mathscr{L}_{V}^{(A)}=\left\langle V_{\mu v} \mathbb{O}_{V}^{\mu v}\right\rangle, \quad \mathbb{O}_{V}^{\mu v}=\frac{F_{V}}{2 \sqrt{2}} f_{+}^{\mu v}+\frac{i G_{V}}{2 \sqrt{2}}\left[u^{\mu}, u^{v}\right]+\frac{c_{1}^{V}}{2}\left(\nabla^{\mu} J_{V}^{v}-\nabla^{v} J_{V}^{\mu}\right) / v^{2},(2
$$

with $\langle\ldots\rangle$ for the matrix trace, $u_{\mu}=i u\left(D_{\mu} U\right)^{\dagger} u$, the combinations $f_{ \pm}^{\mu v}=u^{\dagger} \hat{W}^{\mu v} u \pm u \hat{B}^{\mu v} u^{\dagger}$ of the left and right field-strength tensors $\hat{W}^{\mu v}$ and $\hat{B}^{\mu v}$, respectively, and $U=u^{2}=\exp \left\{i \varphi^{a} \sigma^{a} / v\right\}[21$, 22]. The precise definition of the covariant derivatives $D_{\mu}$ and $\nabla_{\mu}$ can be found in [21,22]. The tensor $J_{V}^{\mu}=-\operatorname{Tr}_{\mathrm{D}}\left\{\xi \bar{\xi} \gamma^{\mu}\right\}$ introduces the fermionic vector current in a covariant way, with $\xi=$ $u \psi_{R}+u^{\dagger} \psi_{L}$ given by the $S U(2)_{R, L}$ doublets $\psi_{R, L}=\frac{1}{2}\left(1 \pm \gamma_{5}\right) \psi$, with $\psi=(t, b)^{T}$ (other SM doublets can be also added [6]) and the Dirac trace $\operatorname{Tr}_{\mathrm{D}}$. The superscript $(A)$ refers to the antisymmetric tensor formulation employed for the spin-1 resonance [15]. The full Lagrangian may contain additional operators not relevant for the form-factors analyzed in this talk [2]. Integrating out $V$ one gets a contribution to the EWET, which at lowest order is given by

$$
\Delta \mathscr{L}_{\mathrm{EWET}}^{\text {from }}=\frac{\left\langle\mathbb{O}_{V}^{\mu v}\right\rangle^{2}}{2 M_{V}^{2}}-\frac{\left\langle\mathbb{O}_{V}^{\mu v} \mathbb{O}_{V \mu \nu}\right\rangle}{M_{V}^{2}}=\underbrace{-i \frac{F_{V} G_{V}}{4 M_{V}^{2}}}_{=i \mathscr{F}_{3} / 2}\left\langle f_{+}^{\mu v}\left[u_{\mu}, u_{v}\right]\right\rangle \underbrace{-\frac{F_{V} c_{1}^{V}}{\sqrt{2} M_{V}^{2}}}_{=\mathscr{F} X \psi^{2}}\left\langle f_{+}^{\mu v} \nabla_{\mu} J_{V v} / v^{2}\right\rangle+\ldots
$$


with the dots standing for other effective operators not relevant in these proceedings. For the Higgsless part, one has $\mathscr{F}_{3}=a_{2}-a_{3}$ in Longhitano's notation of $[9,10]$. In what follows, we will focus on the Higgsless sector and $\mathscr{F}_{3}, \mathscr{F}^{X} \psi^{2}, F_{V}, G_{V}$ and $c_{1}^{V}$ simply represent coupling constants.

The resonance Lagrangian (2.1) provides the vector form-factors of the $L+R$ current into two-Goldstones and into two-fermions [2, 23, 21, 22]:

$$
\mathbb{F}_{\varphi \varphi}^{v}\left(q^{2}\right)=1+\frac{F_{V} G_{V}}{v^{2}} \frac{q^{2}}{M_{V}^{2}-q^{2}}, \quad \quad \mathbb{F}_{f \bar{f}}^{v}\left(q^{2}\right)=1-\frac{\sqrt{2} F_{V} c_{1}^{V}}{v^{2}} \frac{q^{2}}{M_{V}^{2}-q^{2}},
$$

with momentum transfer $q^{\mu}$. The square form-factors $\left|\mathbb{F}_{i i}^{v}(s)\right|^{2}$ contribute to the $S$-parameter at oneloop through the Peskin-Takeuchi sum-rule on the left-right correlator $\Pi_{W^{3} B}$ [19]. If one requires that these form-factors give a ultraviolet-convergent contribution to the sum-rule, they must vanish at $q^{2} \rightarrow \infty$ and one obtains short-distance (SD) constraints [16, 23, 21, 22] and predictions for the LECs $[1,2,16]$ :

$$
F_{V} G_{V}=v^{2} \quad \longrightarrow \quad \mathscr{F}_{3}=\left(a_{2}-a_{3}\right)=-\frac{F_{V} G_{V}}{2 M_{V}^{2}} \quad \stackrel{\mathrm{SD} \text { constr. }}{=}-\frac{v^{2}}{2 M_{V}^{2}} .
$$

For $M_{V}>1.5 \mathrm{TeV}$ one finds the bound

$$
-1.3 \cdot 10^{-2}<\mathscr{F}_{3}=\left(a_{2}-a_{3}\right)<0 .
$$

One can obtain analogous bounds for the LEC $\mathscr{F}^{X} \psi^{2}=v^{2} /\left(2 M_{V}^{2}\right)$ by demanding a similar SD behaviour $\mathbb{F}_{f \bar{f}}^{v}\left(q^{2}\right) \stackrel{q^{2} \rightarrow \infty}{\longrightarrow} 0$ to the fermion form-factor, which would give $\sqrt{2} F_{V} c_{1}^{V}=-v^{2}$.

\section{1 $\mathbb{F}_{\varphi \varphi}^{v}$ form-factor: S-parameter}

The impact of the bosonic form-factor $\mathrm{F}_{\varphi \varphi}^{v}$ on the oblique parameters $S$ and $T$ was studied in a dispersive one-loop resonance analysis [23, 21, 22], where the lightest triplet vector $(V)$ and axial-vector $(A)$ resonances were taken into account. Therein, the contribution from the Goldstone and Higgs absorptive channels was incorporated. In particular the $\mathrm{F}_{\varphi \varphi}^{v}\left(q^{2}\right)$ determined the contribution from the $\varphi \varphi$ and $B \varphi$ cuts to the $S$ and $T$ parameter, respectively [22]. We studied asymptotically-free strongly coupled theories, where $\Pi_{W^{3} B}$ satisfies the two Weinberg Sum Rules (WSRs), and scenarios with weaker ultraviolet (UV) conditions (only the 1st WSR applies) such as Conformal [24] or Walking [25] Technicolour, obtaining the 68\% confidence level determinations [22]:

$$
\begin{aligned}
0.97<\kappa_{W}=M_{V}^{2} / M_{A}^{2}<1, & M_{V}>5 \mathrm{TeV} \quad(1 \text { st \& 2nd WSR) } \\
0.84<\kappa_{W}<1.30, & \left.M_{V}>1.5 \mathrm{TeV} \text { (only 1st WSR, for } 0.5<M_{V} / M_{A}<1\right),
\end{aligned}
$$

where $\kappa_{W}$ denotes the $h W W$ (and $h \varphi \varphi$ ) coupling in SM units $\left(\kappa_{W}^{\mathrm{SM}}=1\right)$.

\section{$2.2 \mathbb{F}_{f \bar{f}}^{v}$ form-factor: $Z \rightarrow f \bar{f}$ anomalous couplings}

The $v_{f}$ and $a_{f}$ constants that parametrize the $Z \rightarrow f \bar{f}$ decay have the form [26],

$$
v_{f}=T_{3}^{f}-2 Q_{f} \sin ^{2} \theta_{W}+\left(\delta g_{R}^{Z f}+\delta g_{L}^{Z f}\right), \quad a_{f}=T_{3}^{f}+\left(\delta g_{R}^{Z f}-\delta g_{L}^{Z f}\right),
$$


with $T_{3}^{t}=+1 / 2, T_{3}^{b}=-1 / 2$, the electric charge $Q_{f}$, the weak angle $\theta_{W}$ and the new physics parametrized through the $\delta g_{R, L}^{Z f}$, given in our low-energy description by

$$
\left|\delta g_{R, L}^{Z f}\right|=\left|\mathscr{F}^{X \psi^{2}}\right| \cos \left(2 \theta_{W}\right) m_{Z}^{2} / v^{2}
$$

in agreement with current bounds of $\mathscr{O}\left(10^{-3}\right)$ [27] for the fermion coupling $\mathscr{F} X \psi^{2} \sim v^{2} /\left(2 M_{V}^{2}\right)<$ $1.3 \cdot 10^{-2}$ that one gets from the previous resonance coupling estimate $\sqrt{2} F_{V} c_{1}^{V}=-v^{2}$, the bound $M_{V}>1.5 \mathrm{TeV}[22]$ and the experimental value $\cos \left(2 \theta_{W}\right) m_{Z}^{2} / v^{2}=0.07$.

\section{Equivalent Proca four-vector representation}

Through an appropriate duality transformation in the generating functional it is possible to rewrite the underlying resonance Lagrangian $\mathscr{L}^{(A)}$ in $(2.1)$ as a Proca Lagrangian $\mathscr{L}^{(P)}$ in terms of four-vector field $\hat{V}_{\mu}$ and its field strength tensor $\hat{V}_{\mu \nu}=\nabla_{\mu} \hat{V}_{v}-\nabla_{v} \hat{V}_{\mu}$. A similar procedure [2, $16,28]$ can be applied to models where the resonances are introduced as gauge fields [29]. In the process, additional non-resonant operators with only light dof are generated, which guarantee a proper UV behaviour. [16, 23, 28]. On-shell, this duality can be read as $V^{\alpha \beta}=\hat{V}^{\alpha \beta} / M_{V}$ and $\nabla_{\rho} V^{\rho \mu}=-M_{V} \hat{V}^{\mu}$. In our particular case, the duality transformation $[2,28]$ changes the antisymmetric tensor Lagrangian (2.1) into

$$
\begin{aligned}
\mathscr{L}^{(A)} \longrightarrow \mathscr{L}^{(P)}= & \left\langle\hat{V}_{\mu v}\left(\frac{f_{\hat{V}}}{2 \sqrt{2}} f_{+}^{\mu v}+\frac{i g_{\hat{V}}}{2 \sqrt{2}}\left[u^{\mu}, u^{v}\right]\right)+\hat{V}_{\mu}\left(\zeta_{\hat{V}} J_{V}^{\mu} / v^{2}\right)\right\rangle \\
& -\left\langle\left(\frac{f_{\hat{V}}}{2 \sqrt{2}} f_{+}^{\mu v}+\frac{i g_{\hat{V}}}{2 \sqrt{2}}\left[u^{\mu}, u^{v}\right]\right)^{2}\right\rangle,
\end{aligned}
$$

with $f_{\hat{V}}=F_{V} / M_{V}, g_{\hat{V}}=G_{V} / M_{V}$ and $\zeta_{\hat{V}}=c_{1}^{V} M_{V}$. In the low-energy limit $p \ll M_{V}$, Eq. (3.1) leads to the same EWET,

$$
\mathscr{L}_{\mathrm{EWET}}=-i \frac{f_{\hat{V}} g_{\hat{V}}}{4}\left\langle f_{+}^{\mu v}\left[u_{\mu}, u_{v}\right]\right\rangle-\frac{f_{\hat{V}} \zeta_{\hat{V}}}{\sqrt{2} M_{V}^{2}}\left\langle f_{+}^{\mu v} \nabla_{\mu} J_{V v} / v^{2}\right\rangle+\ldots
$$

The same agreement is found for the two form-factors previously obtained in (2.3):

$$
\mathrm{F}_{\varphi \varphi}^{v}\left(q^{2}\right)=1+\frac{f_{\hat{V}} g_{\hat{V}}}{v^{2}} q^{2}+\frac{f_{\hat{V}} g_{\hat{V}}}{v^{2}} \frac{q^{4}}{M_{V}^{2}-q^{2}}, \quad \mathrm{~F}_{f \bar{f}}^{v}\left(q^{2}\right)=1-\frac{\sqrt{2} f_{\hat{V}} \zeta_{\hat{V}}}{v^{2}} \frac{q^{2}}{M_{V}^{2}-q^{2}} .
$$

\section{Conclusions}

The EWET couplings can be predicted in terms of resonance parameters; different resonance quantum numbers lead to different patterns for the LECs [1, 15, 17]. Further assumptions about the UV structure of the underlying theory can be used to refine the predictions [1, 22]. In this talk we have provided a couple of examples (oblique parameters $S$ and $T$ and the anomalous $Z f \bar{f}$ couplings) to show that composite resonances with masses of a few $\mathrm{TeV}\left(M_{R} \sim 4 \pi v \approx 3 \mathrm{TeV}\right)$ are compatible with present direct and indirect searches. The $S U(2)_{L} \times S U(2)_{R}$ chiral invariance of the ECLh leads to an appropriate low-energy suppression of tree-level NLO corrections by factors $a_{k} p^{2} / v^{2} \sim p^{2} / M_{R}^{2}$ with respect to the LO prediction, $\mathscr{O}\left(p^{2}\right)[1,15,16]$. Finally, we have shown the equivalence between the antisymmetric tensors $V^{\mu \nu}$ and Proca four-vectors $\hat{V}^{\alpha}$ representations for spin-1 fields [16, 28]. 


\section{References}

[1] A. Pich, I.Rosell, J. Santos and J.J. Sanz-Cillero, [arXiv:1510.03114 [hep-ph]].

[2] A. Pich, I.Rosell, J. Santos and J.J. Sanz-Cillero, in preparation.

[3] S. Weinberg, Physica A 96 (1979) 327.

[4] H. Georgi and A. Manohar, Nucl. Phys. B 234 (1984) 189.

[5] G. Buchalla, O. Catà and C. Krause, Phys. Lett. B 731 (2014) 80; J. Hirn and J. Stern, Eur.Phys.J. C 34 (2004) 447; JHEP 0409 (2004) 058.

[6] F.-K. Guo, P. Ruiz-Femenía and J.J. Sanz-Cillero, Phys.Rev. D 92 (2015) 7, 074005.

[7] J. Gasser and H. Leutwyler, Annals Phys. 158 (1984) 142; Nucl. Phys. B 250 (1985) 465.

[8] G. Buchalla, O. Catà and C. Krause, Nucl. Phys. B 880 (2014) 552.

[9] A. C. Longhitano, Phys. Rev. D 22 (1980) 1166; Nucl. Phys. B 188 (1981) 118; T. Appelquist and C. W. Bernard, Phys. Rev. D 22 (1980) 200; F. Feruglio, Int.J.Mod.Phys. A 8 (1993) 4937.

[10] M.J. Herrero and E. Ruiz Morales, Nucl. Phys. B 418 (1994) 431.

[11] G.F. Giudice et al., JHEP 0706 (2007) 045.

[12] R. Alonso et al., Phys.Lett. B722 (2013) 330.

[13] B. Grinstein and M. Trott, Phys. Rev. D 76 (2007) 073002.

[14] R. Alonso, E.E. Jenkins and A.V. Manohar, [arXiv:1511.00724 [hep-ph]].

[15] G. Ecker et al., Nucl. Phys. B 321 (1989) 311.

[16] G. Ecker et al., Phys. Lett. B 223 (1989) 425.

[17] Oscar Catà, Eur. Phys. J. C 74 (2014) 8, 2991 [arXiv:1402.4990 [hep-ph]].

[18] S. Weinberg, Phys. Rev. Lett. 18 (1967) 507; C. W. Bernard et al., Phys. Rev. D 12 (1975) 792.

[19] M. E. Peskin and T. Takeuchi, Phys. Rev. D 46 (1992) 381; Phys. Rev. Lett. 65 (1990) 964.

[20] S. J. Brodsky and G. R. Farrar, Phys. Rev. Lett. 31 (1973) 1153; G. P. Lepage and S. J. Brodsky, Phys. Rev. D 22 (1980) 2157.

[21] A. Pich, I. Rosell and J.J. Sanz-Cillero, JHEP 1208 (2012) 106 [arXiv:1206.3454 [hep-ph]].

[22] A. Pich, I. Rosell and J. J. Sanz-Cillero, Phys. Rev. Lett. 110 (2013) 181801; JHEP 1401 (2014) 157.

[23] R. Barbieri et al., Phys.Rev. D 78 (2008) 036012.

[24] A. Orgogozo and S. Rychkov, JHEP 1203 (2012) 046.

[25] T. Appelquist and F. Sannino, Phys. Rev. D 59 (1999) 067702.

[26] A. Pich, [arXiv:1201.0537 [hep-ph]].

[27] K. Agashe et al., Phys.Lett. B 641 (2006) 62; A. Efrati, A. Falkowski and Y. Soreq, JHEP 1507 (2015) 018.

[28] J. Bijnens and E. Pallante, Mod.Phys.Lett. A 11 (1996) 1069; K. Kampf, J. Novotny and J. Trnka, Eur.Phys.J. C 50 (2007) 385.

[29] M. Bando, T. Kugo and K. Yamawaki, Phys. Rept. 164 (1988) 217; M. Harada and K. Yamawaki, Phys.Lett. B 297 (1992) 151; Phys.Rep. 381 (2003) 1; R. Casalbuoni et al, Int.J.Mod.Phys. A 4 (1989) 1065; R. Casalbuoni et al., Nucl.Phys. B 409 (1993) 257. 